\title{
Representology in the System of Modern Epistemology
}

\author{
Vladimir Inozemtsev \\ Moscow State Technical University named after N.E. \\ Bauman \\ (National Research University) \\ Moscow, Russia \\ E-mail: inozem_63@mail.ru \\ Vitaly Ivlev \\ Moscow State Technical University named after N.E. \\ Bauman \\ (National Research University) \\ Moscow, Russia \\ E-mail: vitalijivlev@yandex.ru
}

\begin{abstract}
The article is dedicated to the development of representology as a conceptual formalization of the problem of representation of knowledge in modern science. The work defines the subject, main sections and problems of suggested by the author's concepts of philosophical, cognitive and computer representology, as well as concepts of AI epistemology and computer epistemology.
\end{abstract}

Keywords-representology; problem of representation of knowledge; problem of computer representation of knowledge; discursive and engineering approaches to knowledge; cognitive sciences; computer sciences; artificial intelligence (AI); epistemological content (AI); epistemology of AI; computer epistemology

\section{INTRODUCTION}

In the modern era of formation and development of information society, there is a need in further study of knowledge itself, which until the middle of the 20th century acted primarily as a subject of the theory of cognition. In recent decades, knowledge becomes the subjects of cognitive and computer sciences, and interdisciplinary area of researches with metaphorical title "artificial knowledge" (AI).

In knowledge, the contents are distinguished along with the form of its expression and presentation, which is designated with the term "representation". Representation as a poly-semantic concept currently finds broad application in different scientific disciplines and means representation of one object by another object. The concept of representation was initially approved in cognitive sciences that were engaged in the study of structures and processes of cognitive activity in general and representation in particular. The term "representation" in cognitive sciences usually means mental representations, whereby coding and amassing of sensory information in the brain takes place.

\author{
Marina Ivleva \\ Moscow Institute of Psychoanalysis \\ Moscow, Russia \\ E-mail: marinanonna@yandex.ru
}

\author{
Mikhail Oseledchik \\ Department of Philosophy and Social Sciences \\ Moscow State University of Printing named after I. Fedorov \\ Moscow, Russia \\ E-mail: balu13@yandex.ru
}

The term "representation" from cognitive sciences goes from computer sciences to AI, where the new concept of "representation of knowledge" is formed. In these scientific disciplines, this concept is used for representation of socalled computer knowledge. Through the concept of "computer knowledge", AI designate a set of statements about domain objects, their properties, relations, events, processes and patterns of structure of functioning of these areas, as well as the rules of derivation of some statements from others. In this article, we'll use the concept of "computer knowledge" in typical for AI understanding due to its widest dissemination in this interdisciplinary area of research, though this concept is not very usual to philosophers and not always accepted. Computer knowledge in AI includes knowledge of experts and objectified knowledge contained in various documents and natural language texts. They are codified, formalized and imprinted in sign systems specific to AI (computer models and programs, programming languages).

Finally, in the $1980 \mathrm{~s}$, the term of "representation of knowledge" starts moving into the philosophical lexicon. The phrase "representation of knowledge" in modern philosophy means the concept of results of multiple forms of cognitive activity with different natures, their codifications and imprints in sign systems through intermediaries such as models, natural and artificial languages as well as various logical and mathematical systems. Research of representation of knowledge in this sense involves finding out by which structures and in which forms the knowledge is represented in sign systems in particular and languages (including artificial) in general. 
II. THE PROBLEM OF REPRESENTATION OF

KNOWLEDGE: DISCURSIVE AND ENGINEERING APPROACHES TO THE STUDY OF KNOWLEDGE

During the 1970s, when the term "representation of cognition" starts to actively apply, the problem of computer representation of knowledge is formed. On one side, the essence of this problem is due to dissonance between existing expert non-formalized knowledge of patterns of structure and functioning of subject areas. From the other side, the need of fixation, codification, formalization of this non-formalized knowledge and its imprinting in computer memory via sign systems (computer models, programs and programming languages) in the form of computer knowledge. The problem of computer representation of knowledge is solved by creation of concepts of computer representation of knowledge (logical, network, framing), each of which represents a set of relevant models and languages of computer representation of knowledge.

The problem of computer representation of knowledge represents the modern technical option of common problem of representation of knowledge. On one hand, the essence of the latter is due to disparity between the existence of nonformalized (substantial) knowledge about the world that is present in each subject of cognitive activity, and, the need for formalization and representation of this knowledge via sign (linguistic, mathematical, logical) systems is on the other hand. A common problem of representation of knowledge was, until recently, in the shadow of other, more significant philosophical problems, and only separate aspects of this problem get analyzed.

In the majority of philosophical systems up to the latter decades of the 20th century, the term of representation of knowledge didn't have a pronounced categorical status, and the problem of representation of knowledge in philosophical literature is usually not articulated until 1980s. There is a paradoxical situation. On one hand, the phenomenon of representation of knowledge is broad, complex and its research just in historical and philosophical tradition requires a lot of effort. On the other hand until recently, philosophical works do not attempt complex philosophical and methodological analysis of this phenomenon and associated problem of representation of knowledge, as well as the research of correlation of this problem with other philosophical problems and categories. The problem of representation of knowledge gains autonomy and gets actualized in the recent decades due to intensive development of computer and cognitive sciences and formalization of its variety in the problem of computer knowledge.

We'll allocate discursive and engineering approaches as the fundamental approaches to the study of knowledge. In the spotlight of the discursive approach to the study of knowledge are the problems of the essence of knowledge, the method of its existence, the difference between knowledge and quasi-knowledge, verity of knowledge, its structural organization, and the ratio of personal and impersonal knowledge. The engineering approach to knowledge concentrates on the solution of instrumental and the technological problems of acquisition, representation, processing and translation of knowledge, as well as the analysis of different methods of working with knowledge. Discursive and engineering approaches to the study of knowledge arise almost simultaneously during the era of antique classic yet from that point on develop irregularly. Each of these approaches to the study of knowledge can be distributed to the corresponding approaches to representation of knowledge. Because of this, it's possible to talk about discursive and engineering approaches not only to the study but to the representation of knowledge.

Until the mid-20th century, the dominant approach in philosophy and science was discursive approach to study and representation of knowledge. This doesn't mean that until then the engineering approach to knowledge did not develop. However its dawn begins in the middle of the 20th century, peaks in 1960s - 1980s, and is driven by the rapid improvement of the means of computer technology and the development of intellectual information technologies in terms of information and computer resolution. In recent decades, this approach becomes more prevalent in research and representation of knowledge in modern science.

The traditional engineering approach to the study of knowledge is embodied in computer science and the area of AI during the latter third of the 20th century in the engineering cybernetic approach to the study and representation of knowledge, which is a kind of systematic approach to the research of the development of engineering and technology. Engineering and cybernetic approach involves the construction and use via technical means such sign systems by which computer knowledge is acquired, represented, translated, updated and summarized, as well as the conclusion on computer knowledge is output. As the sign systems in AI are the models and languages of computer representation of knowledge being the various solutions of the problem of computer representation of knowledge.

The problem of computer representation of knowledge forms the core of epistemological content of $A I$, which represents a set of problems and methods for their solution associated with transformation in methods of production of computer knowledge, their fixation, adaptation and use. The emergence of these problems is caused by intensive development and application of intellectual and other modern information technologies as well as the reflection of computer knowledge. The epistemological content of AI includes such areas of the problems of AI as manipulation of knowledge, its updating, generalization and classification, acquisition of knowledge, computer cognition and computer comprehension and some others.

It is necessary to distinguish between epistemological content of AI in both a broad and narrow sense. The epistemological content of AI in a narrow sense means summation of epistemological ideas, problems and their solutions created by the computer representation of knowledge and manipulation of knowledge, with which the research of different methods of reasoning and conclusions of various computer knowledge from other. The epistemological content of AI in a broad sense means 
epistemological problems and their solutions, which alongside the computer representation of knowledge and the manipulation of knowledge also include the acquisition of knowledge, their classification, updating and generalization, computer perception and computer comprehension.

\section{REPRESENTOLOGY AND ITS EPISTEMOLOGICAL STATUS}

Let's turn to constitution of reflection of the problem of representation of knowledge in philosophy, computer and cognitive science in new, independent epistemological concepts. To refer these concepts, we'll use previously introduced by the author of this article in a number of previous works [1-4] term "representology", which refers to a set of concepts investigating the problem of representation of knowledge in different scientific disciplines and interdisciplinary areas of study.

The term "representation of knowledge" appears, in the first place, due to the advancement of computer sciences and computer methods of representation of information. With this, this term can be used in relation to any method of representation of information (primarily of knowledge) via the usage of sign systems that are used in different areas of human activity. Based on the disambiguation of the term "representation of knowledge" and its widespread use in such sciences as philosophy, cognitive and computer science, we distinguish in the structure of representology the sections of philosophical representology, cognitive representology and computer representology. Let's try to consider in the most general contours the subject and the main problems of such becoming epistemological concepts as philosophical, cognitive and computer representology as well as related to them concepts of computer representology and epistemology of artificial intelligence (AI-epistemology).

The concept of representology is filled with specific content during the study of interrelationship of philosophical methodology and epistemology with methodology of computer and cognitive science. The successful development of computer and cognitive science requires not only special attention to philosophical problems of these sciences but an elaborate and conceptualized methodological and epistemological base, special epistemological conception of methods of providing information and, especially, of knowledge via sign systems. This place can be claimed by the concepts of philosophical, cognitive and computer representology and the concepts of computer epistemology and AI-epistemology.

The object of the study in representology is the system of modern scientific cognition. The subject of representology (including philosophical, cognitive and computer representology) is representation of knowledge as a complex structured phenomenon and its epistemological status. The main objective of these sections of representology is the research of specifics and solutions of the problem of representation of knowledge in corresponding scientific disciplines and interdisciplinary areas of research. These concepts differ both from cognitive and computer science and are from traditional epistemology even though they're closely related and closely cooperate.

The sections of representology face a variety of problems:

- the analysis of specifics of representation of knowledge in philosophy, computer science and AI, in cognitive science;

- the research of representation of standardized forms of knowledge embodied in natural language texts and representation of non-traditional forms of human knowledge (implicit, universalized, personal, subjectified);

- the study of ways and means of the construction of an integrated model of representation of knowledge;

- the analysis of genesis and evolution of philosophical teachings of representation of knowledge, ideas about representation of knowledge in cognitive science, concepts of computer representation of knowledge (logical, network, framing).

In terms of content representology, cognitive and computer science, engineering of knowledge and epistemology are closely interrelated. Without a holistic methodological models of representation of knowledge, the development of cognitive and computer science is severely impeded. At the same time, representology should represent the reflection of the front line of these sciences. Engineering of knowledge, the epistemological component content of AI in a broad sense, acts as a peculiar "touchstone" for computer representology.

Look at the different sections of representology. The concept of philosophical representology represents the modern epistemological concept in which the theoretical reconstruction of conceptual formalization of the problem of representation of knowledge in historical and philosophical tradition occurs. Within the concept of philosophical representology, there's the study of the establishment and development of the reaching of philosophical representation of knowledge, which represent different solutions to this problem occurring in the history of epistemology. The problem of representation of knowledge can be isolated in the context of epistemological concepts, starting with antique philosophical teachings up to the modern epistemological theories. The philosophical representology uses as discursive, so partly engineering approach to the research and representation of knowledge.

Historical-philosophical reconstruction of conceptual formalization of the problem of representation of knowledge is realized according with the two most important attitudes. Foremost, the problem of representation of knowledge is interpreted as an imminent component of historically varied and successive epistemological concepts. Secondly, it's seen in the aspect of its essential characteristics, expressed in that this problem serves to hold various methods of representation of knowledge through the use of sign systems in a set of sciences (philosophy, computer and cognitive science). This research perspective allows to implement the necessary analysis of genesis and evolution of the problem of 
representation of knowledge in historical and philosophical tradition, and it created the prerequisites for correlation of the solution of this problem in philosophy with the specifics of its solution in cognitive and computer science to philosophical and methodological analyses of which the next sections of the general representology - cognitive and computer representology - are dedicated.

On the design of the conceptual content of the modern technical version of the problem of representation of knowledge - the problem of computer representation of knowledge - during 60s-70s of the 20th century is primarily influenced by the breakthroughs in the area of AI, as well as the results obtained in cognitive sciences [5-11]. The attempts of computer thinking simulation from the genesis of this idea give an impetus to the researches in cognitive science and, primarily, in cognitive psychology, aimed at the study of cognitive activity, and thereafter to the researches in cognitive linguistics and cognitive psycholinguistics [12].

It should be noted that the results, received in AI and computer science in general affect the cognitive science, and the achievements in cognitive science, in turn, have an impact on the new studies in the areas of AI and computer representation of knowledge. At first, the researches in cognitive psychology in 50s-60s of the 20th century were highly influenced by the informative approach and the computer metaphor. The emergence of which is caused by the development of information and computer technologies, cybernetic movement and the successes with AI. In accordance with the computer metaphor, the brain and the mind of a human is compared to a computer, and cognitive processes are considered by an analogy with computer information processing. The results, obtained in computer science and AI, impacts throughout its existence the cognitive science.

On the other hand, from the end of the 1970s to the middle of the 1980s, the successes and achievements of cognitive science and, in particular, of cognitive psychology, are beginning to have an impact on the studies in the areas of AI and computer representation of knowledge. This applies primarily to the formation, under the computer representation of knowledge, of the concepts of network and framing. These concepts appear as constructs of cognitive psychology, and frames and semantic networks are referred to, in the moment of their creation, as cognitive structures [13]. Later on they are viewed as structures of computer representation of knowledge $[14,15]$. However, it should be remembered that both frame and network concepts of representation of knowledge are based on the concepts of the structures of human cognition and memory, developed in cognitive psychology. However, both of these concepts of computer representation of knowledge, thus far, have independent meanings as psychological and epistemological concepts and are used in solutions beyond computer representation of knowledge.

On this basis, the following section of general representology shall be named the concept of cognitive representology. This concept studies the representation of knowledge in cognitive sciences, including in cognitive psychology, cognitive linguistics, cognitive psycholinguistics, evolutionary theory of cognition, and ecological epistemology. Cognitive representology uses both discursive as well as engineering approaches to the study and representation of knowledge.

The problem of computer representation of knowledge becomes the object of consideration of the concept of computer representology. Computer representology explores the key aspects of computer representation of knowledge (logical, network, framing), developed in the later decades in AI [16-23]. These concepts represent different solutions to the discussed previously problem of computer representation of knowledge and, in the narrow sense, form the core of epistemological contents of AI.

\section{COMPUTER EPISTEMOLOGY AND EPISTEMOLOGY OF ARTIFICIAL INTELLIGENCE}

Computer representology is one of the leading areas of the modern postnonclassical concept of epistemology in computer science (or the concept of computer epistemology), alongside several other problems is considered an earlier problem of computer representation of knowledge is studied. As the most important factor of constructing of the new epistemological paradigm and methodology of study and representation of knowledge in modern science (especially in computer science and AI), the series of radical transformations in engineering and technology, primarily information and communication, which began in the middle of 20th century and constantly gathered pace. With time, this set of qualitative modifications and transformations receives the name of information and computer revolution.

One of the consequences of information and computer revolution was the improvement of intellectual information technologies, which necessitates the new outlook at natural intelligence and the possibility of its computer modeling [24]. There is a circle of problems and ideas related to the new section of representology, named in the article AIepistemology (epistemology of artificial intelligence). To solve the problems of AI-epistemology, the efforts of classical academic epistemology are not enough anymore.

During the $1950-1960$ s researchers are turning to the solution of the problems of philosophical understanding of information and intelligence: the specifics of the computer knowledge that grow out of computer data in the process of development of information and communication technologies. AI-epistemology studies computer knowledge from the point of representation, transformation and translation of information, and the conversion of this information into computer knowledge. This epistemology appeals to the analysis of the new epistemological problems, including the problems of representation, manipulation, acquisition, adjunction, generalization and classification of computer knowledge, as well as the problems of computer perception and computer understanding.

Intellectual activity in AI-epistemology refers to the realization of certain cognitive structures and processes. Cognitive processes in new epistemology refer to the processes of computer transformation of information, and 
thought is defined as a computational process. Due to this, in AI-epistemology appear and are studied new ideas, approaches and concepts that differ from classical epistemology. AI-epistemology considers computer knowledge as the highest form of representation of information in AI. On this basis the goals, objectives and accents, specific to classical epistemology, are radically changed in AI-epistemology. At the same time, AIepistemology and classical epistemology have some areas of intersection.

AI-epistemology represents one of the most important sections of computer epistemology. Among the problems of computer epistemology, along the previously mentioned problem of AI-epistemology, are also the problems of comprehension of the essence of information; of study of mechanisms of storage, translation and acquisition of information in computer systems (including computer knowledge); of figuring out if computers can think; of analysis of concepts of engineering of knowledge and many others. The presence of the new problems and the development if their solutions turn the computer epistemology into a new, independent section of the modern postnonclassical epistemology.

\section{CONCLUSION}

Let us consider the correlation of subjects, problems and areas of application of philosophical representology, computer epistemology, AI-epistemology and computer representology. Philosophical representology is a section of epistemology in which the study of the general problem of representation of knowledge occurs. This representology covers the circle of problems primarily related to discursive and somewhat engineering approach to the study and representation of knowledge. Philosophical representology studies formation and development of different concepts of philosophical representation of knowledge. They represent the teachings of representation of knowledge which took place in historical philosophical tradition from antique to modern time.

Computer epistemology is a section of new epistemology (primarily, postnonclassical), dedicated to the analysis of the problems, caused by the development of the modern technological artifacts, reprocessing the knowledge (computers) and their increasing influence at cognitive activity.

As one of the leading areas of computer epistemology stands epistemology of artificial intelligence (AIepistemology). It's a section of postnonclassical epistemology that possesses a specific set of ideas, problems and their solutions, generally related, in a broad sense, to the epistemological contents of AI. One of the most important among them is the problem of computer representation of knowledge.

Finally, computer representology is a section of AIepistemology and by that of computer epistemology in general. It studies the concepts of computer representation of knowledge that represent different approaches to the solution of problem of computer representation of knowledge.

\section{REFERENCES}

[1] Inozemtsev V.A. Representation of knowledge in modern science philosophical and methodological analysis. M.: MSTU "MAMI". 2009. $234 \mathrm{p}$.

[2] Inozemtsev V.A. Logical and epistemological study of artificial intelligence: the phenomenon of computer representation of knowledge. M.: "ITO SEMRIK". 2014. 160 p.

[3] Inozemtsev V.A. Epistemological content of artificial intelligence. M.: "ITO SEMRIK" LLC, 2015. 157 p.

[4] Inozemtsev V.A. Deductive logic to solve the problem of computer representation of knowledge // Proceedings of MSTU "MAMI". M., 2014. \#1 (19), V. 5. P. 121 - 126.

[5] Baksansky O.E., Kucher E.N. Cognitive science. From knowledge to action. M.: Editorial-URSS. 2005. 182 p.

[6] Belyanin V.G. Introduction to psycholinguistics. M.: Moscow State Linguistic University. 1999. 126p.

[7] Minsky M. Frames for knowledge representation. M.: Mir. 1979. 151 p.

[8] Neisser U. Cognitive Psychology. M.: Trivola. 2000. 467 p.

[9] Solso R. Cognitive Psychology. M.: Trivola. 1996. 598 p.

[10] Boden M. Artificial intelligence in psychology. Cambridge. (Mass.) MIT Press. 1988. 188p.

[11] Pylyshyn Z. Computation and cognition. Forward a foundation for cognitive science. Cambridge. (MAss.). MIT Press. 1985. 292 p.

[12] Belyanin VG Introduction to psycholinguistics. M.: Moscow State Linguistic University. 1999. 126 p.

[13] Minsky M. Frames for knowledge representation. M.: Mir. 1979. 151

[14] Winograd T. Frame representation and declarative-procedural controversy // Representation and understanding. N.-Y.: Academic Press. 1975. P.185-210.

[15] Introduction and use of knowledge / Ed. H.Ueno, M.Isudzuka. M. Mir. 1989. 220 p.

[16] Inozemtsev V.A. Representation of knowledge in modern science: philosophical and methodological analysis. M.: MSTU "MAMI". 2009. 234 p.

[17] Inozemtsev V.A. Deductive logic to solve the problem of computer representation of knowledge // Proceedings of MSTU "MAMI". M., 2014. \#1 (19), V. 5. P. 121 - 126.

[18] Logical approach to artificial intelligence. M.: Mir.1990. 233 p.

[19] Pospelov D.A. Logico-linguistic models in management. M. Energoatomisdat. 1981. $231 \mathrm{p}$.

[20] Introduction and use of knowledge / Ed. H.Ueno, M.Isudzuka. M. Mir. 1989. 220 p.

[21] Philosophy of Artificial Intelligence. Proceedings of All-Russia interdisciplinary conference. M.: IP RAS. 2005. 399 p.

[22] Orlowska E., Pawlak Zd. Logical foundations of knowledge representation. Warsaw. IPI. 1984. 106 p.

[23] Winograd T., Florens W. Understanding computers and cognition. N.-Y.: Academic Press. 1987. 209 p.

[24] Arbib M. Metaphorical brain. M.: Editorial-URSS. 2004. 295 p. 